\title{
Diazepam, em dose única, inibe a migração celular, a estimulação macrofágica e a atividade de TNF- $\alpha$ na reação inflamatória aguda induzida por LPS em camundongos
}

\author{
Diana Amaral Monteiro', Iracilda Zeppone Carlos², Fábio Gonçalves Pinto ${ }^{1 *}$ \\ ${ }^{1}$ Departamento de Morfologia e Patologia,, Universidade Federal de São Carlos, ${ }^{2}$ Departamento de Análises \\ Clínicas, Faculdade de Ciências Farmacêuticas, Universidade Estadual Paulista "Júlio de Mesquita Filho"
}

*Correspondência:

F. G. Pinto

Departamento de Morfologia e

Patologia

Centro de Ciências Biológicas e da Saúde

Universidade Federal de São Carlos Rodovia Washington Luiz, km 235 13565-905 - São Carlos - SP, Brasil. E-mail: fgpinto@.ufscar.br
Os benzodiazepínicos estão entre as drogas mais freqüentemente prescritas em razão de suas propriedades ansiolíticas. O objetivo deste trabalho foi avaliar a influência do diazepam sobre a resposta inflamatória peritoneal aguda induzida por lipopolissacarídeo. Para tanto, camundongos Swiss foram tratados com diazepam (1 ou $10 \mathrm{mg} / \mathrm{kg}$ de peso), em dose única, por via subcutânea, uma hora antes do desafio intraperitoneal com lipopolissacarídeo bacteriano. Após 16 horas do desafio, os animais foram sacrificados, coletandose os lavados peritoneais para determinação do número total de células e das subpopulações de mononucleares e polimorfonucleares, além da atividade de TNF- $\alpha$ e da porcentagem de macrófagos espraiados. Observou-se que o tratamento com diazepam, nas doses de 1 ou $10 \mathrm{mg} / \mathrm{kg}$, reduziu significativamente a porcentagem de macrófagos estimulados por LPS e a liberação de TNF- $\alpha$ independente de estímulo. Houve também significativa redução da migração de leucócitos nos animais estimulados com LPS e tratados com $10 \mathrm{mg} / \mathrm{kg}$ de diazepam em relação aqueles não tratados. Concluímos que a administração do diazepam, em dose única, pode influenciar significativamente o influxo celular, a estimulação de macrófagos e a atividade de TNF- $\alpha$ na resposta inflamatória aguda induzida por LPS em camundongos, com possiveis implicações na eficiência da resposta anti-infecciosa.
Unitermos

- Diazepam

- Lipopolissacarídeo

- Resposta inflamatória aguda

- TNF- $\alpha$

- Migração celular/inibição

- Macrófagos/estimulação

\section{INTRODUÇÃO}

Diversas drogas são capazes de deprimir e modular o sistema imune, dentre as quais estão os benzodiazepínicos (BZDs), tais como: diazepam, midazolam e alprazolam (Schlumpf et al., 1990, 1994; Silva, Palermo-Neto, 1999; Zavala, 1997). Os BDZs apresentam propriedades miorrelaxantes, ansiolíticas e sedativas devido à sua alta afinidade com sítios específicos de ligação no sistema nervoso central - os chamados receptores centrais de BZD (RCBs). Desta maneira, os BZDs constituem uma das classes de drogas mais freqüentemente prescritas e consumidas no Brasil, Europa e EUA (Ruiz et al., 1993).

Receptores periféricos de BZDs (RPBs) foram identificados em tecidos endócrinos, fígado, rins, pulmão e testículos (Gavish et al., 1999; Ruff et al., 1985; Wang et al., 
1984) além de células como macrófagos, linfócitos, neutrófilos (Papadopoulos et al., 1990; Zavala, 1997). Alguns estudos demonstraram que os RPBs participam de algumas funções básicas na fisiologia celular, como na esteroidogênese, no crescimento e diferenciação celular, na quimiotaxia e na modulação do sistema imune (Hirsch et al., 1989; Papadopoulos et al., 1990; Schreiber et al., 1993; Zavala et al., 1985).

A densidade de RPBs nas células do sistema imune, é maior que a concentração destes receptores no sistema neuroendócrino (Zavala et al., 1990), o que poderia explicar a ação imunomoduladora de alguns benzodiazepínicos, como a redução da resistência às infecções, redução do burst oxidativo e da fagocitose por macrófagos, inibição da quimiotaxia de células inflamatórias e diminuição da produção do ânion superóxido e de algumas interleucinas (Finnerty et al., 1991; Galdiero et al., 1995; Lazzarini et al., 2001, 2003; Massoco, Palermo-Neto, 1999, 2003; Zhang, Morrison, 1993).

Por outro lado, endotoxinas como os lipopolissacarídeos (LPS) bacterianos têm participação importante em diversos processos infecciosos, sendo potentes estimuladores da produção de citocinas por macrófagos e monócitos (Amura et al., 1997).

Neste sentido, embora o potencial modulador dos BZDs sobre a resposta imune e inflamatória tenha sido comprovado experimentalmente após tratamentos de média e longa duração, poucos trabalhos investigaram seus efeitos agudos sobre a resposta do hospedeiro em agressões induzidas por endotoxinas. Portanto, o objetivo do presente trabalho foi avaliar a influência do diazepam (um BZD extensamente utilizado como ansiolítico), em dose única, sobre o influxo inflamatório, a porcentagem de macrófagos estimulados e a atividade de TNF- $\alpha$ em modelo experimental de peritonite aguda induzida por LPS em camundongos.

\section{MATERIAL E MÉTODOS}

Foram utilizados 60 camundongos Swiss, fêmeas, com 2 meses de idade e aproximadamente $30 \mathrm{~g}$, obtidos no Biotério Central da Universidade Federal de São Carlos (UFSCar). Durante o período experimental, os animais foram alojados em caixas de plásticos no Biotério de Experimentação do Departamento de Morfologia e Patologia, com temperatura controlada e ciclo luz-escuro de $12 \mathrm{~h}$, recebendo ração comercial e água ad libitum.

\section{Tratamento dos animais}

Os animais receberam diazepam (Valium ${ }^{\circledR}$ injetável), por via subcutânea, nas doses de $1 \mathrm{mg} / \mathrm{kg}$ de peso (DZ1) ou $10 \mathrm{mg} / \mathrm{kg}$ de peso (DZ10), num volume de $0,2 \mathrm{~mL}$ ou apenas seu diluente (propilenoglicol a $40 \% \mathrm{em}$ solução salina estéril), utilizado como controle (C). Uma hora após o tratamento, 30 animais foram inoculados por via intraperitoneal com $20 \mu \mathrm{g} /$ animal de lipopolissacarídeo de Escherichia coli sorotipo 0111:B4 (SIGMA L-2630), formando os grupos LPS-C ( $\mathrm{n}=10)$, LPS-DZ1 $(n=10)$, LPS-DZ10 $(n=10)$ e 30 animais receberam solução salina estéril (SSE) via intraperitoneal de maneira a se obter os grupos SSE-C $(n=10)$, SSE-DZ1 $(n=10)$, SSE-DZ10 $(n=10)$.

\section{Obtenção do lavado peritoneal}

Após 16 horas do desafio, os animais foram sacrificados por inalação de éter etílico e fixados em decúbito dorsal em suporte de dissecção para a coleta do lavado peritoneal. O lavado foi obtido seguindo a metodologia descrita por McCarron et al. (1984). Resumidamente, a parede abdominal foi exposta e submetida à desinfecção com álcool 70\%. Em seguida, a cavidade peritoneal foi injetada com $5 \mathrm{~mL}$ de solução salina estéril. Após massagem cuidadosa, a parede abdominal foi seccionada e o fluido peritoneal aspirado com pipeta Pasteur plástica descartável. O material obtido foi centrifugado a $2000 \mathrm{rpm}$ durante 10 minutos e os sobrenadantes aliquotados e estocados a $-20{ }^{\circ} \mathrm{C}$ até o momento das análises. As células foram ressuspensas em SSE para contagem total e diferencial de células e para o teste de espraiamento.

\section{Contagem total e diferencial das células do lavado peritoneal}

A contagem total e diferencial das células recolhidas no lavado peritoneal foi realizada, adaptando-se a coloração de Randolph (Brattig et al., 1993). Alíquotas de $100 \mathrm{~mL}$ da suspensão de células de cada amostra foram misturadas a $20 \mu \mathrm{L}$ de floxina $(0,3 \%$ em propilenoglicol) e $20 \mu \mathrm{L}$ de biebrich $(0,4 \%$ em propilenoglicol $)$ e mantidas em repouso por 3 minutos. Em seguida, foram adicionados $150 \mu \mathrm{L}$ de azul de metileno ( $0,4 \%$ em propilenoglicol) e $20 \mu \mathrm{L}$ de cristal violeta $(0,2 \%$ em propilenoglicol). Após agitação, $10 \mu \mathrm{L}$ desta mistura foram utilizados para a contagem celular em câmara hemocitométrica de Newbauer. Em cada amostra foram contadas, no mínimo, 100 células, estabelecendo-se a porcentagem de mononucleares $(\mathrm{MN})$ e polimorfonucleares (PMN). O número total de células no lavado peritoneal foi calculado após a aplicação de fatores de correção de diluição e volume. 


\section{Determinação do Fator de Necrose Tumoral (TNF- $\alpha$ )}

A quantificação do TNF- $\alpha$ foi realizada utilizando-se a técnica de ensaio biológico, a qual se baseia na propriedade do TNF- $\alpha$ de lisar certas linhagens de células tumorais (Carlos et al., 1994). Foram usadas células tumorais de linhagem L929, sensíveis ao TNF- $\alpha$ ajustadas na concentração de $4 \times 10^{5}$ células $/ \mathrm{mL}$ e $100 \mu \mathrm{L}$ desta suspensão, foram adicionados a cada poço de uma microplaca estéril e incubadas por 18 horas a $37^{\circ} \mathrm{C}$, em atmosfera com $5 \%$ de $\mathrm{CO}_{2}$. Após a incubação, o meio de cultura foi trocado por $100 \mu \mathrm{L}$ de RPMI, contendo actinomicina $\mathrm{D}$ na concentração de $1 \mu \mathrm{g} / \mathrm{mL}$ e foram adicionados $100 \mu \mathrm{L}$ de cada amostra do lavado peritoneal. Após 24 horas de incubação, o sobrenadante foi descartado e as células coradas com cristal violeta $(0,2 \%$ em metanol a $20 \%)$ por 30 minutos em temperatura ambiente. Em seguida, o excesso de corante foi retirado por lavagem em água corrente e $0,1 \mathrm{~mL}$ de lauril sulfato de sódio a $1 \%$, foi adicionado a cada poço para solubilizar o corante retido nas células. A leitura da reação foi realizada sob filtro de $490 \mathrm{~nm}$ em leitor de ELISA (Multiskan Ascent, Labsystems) e comparada à curva-padrão de TNF- $\alpha$ recombinante. A absorbância obtida é diretamente proporcional à quantidade de células vivas e inversamente proporcional à quantidade de TNF- $\alpha$ presente na amostra.

\section{Espraiamento de macrófagos}

A porcentagem de macrófagos peritoneais estimulados foi determinada através da técnica descrita por Rabinovitch et al. (1977), adaptada por Pinto (2000). O teste de espraiamento reflete o estado de estimulação de macrófagos e se fundamenta na propriedade que essas células têm de se aderirem ao vidro e apresentarem espraiamento quando estimuladas. Alíquotas de $100 \mu \mathrm{L}$ de cada suspensão celular do lavado peritoneal foram distribuídas sobre lâminas de vidro e deixadas em repouso por 15 minutos à temperatura ambiente. A seguir, as lâminas foram lavadas com solução salina estéril para a remoção das células não aderidas e, então incubadas em meio de cultura 199 (CULTILAB) durante 1 hora à temperatura de $37^{\circ} \mathrm{C}$. Após a incubação, as lâminas foram lavadas em solução salina estéril e as células aderidas foram fixadas com solução de glutaraldeído a 2,5\% por 5 minutos. Após a fixação, as lâminas foram coradas pela hematoxilina e eosina. Em cada lâmina foram contados no mínimo 100 macrófagos, determinando-se o número e porcentagem de macrófagos espraiados. Todas as análises foram realizadas em duplicata.

\section{Análise estatística}

Os resultados foram analisados estatisticamente, buscando identificar diferenças significativas entre os grupos e subgrupos. Para análise dos dados obtidos no teste de espraiamento, os resultados foram analisados através da comparação de medianas e utilizou-se o teste de Dunn nãoparamétrico. Os demais parâmetros foram expressos como médias \pm erro padrão da média e analisados, aplicando-se análise de variância ANOVA e o teste de Tukey Kramer de comparações múltiplas para $\mathrm{p}<0,05$, sempre que o teste de normalidade de Kolmogorov-Smirnov, confirmava a distribuição normal das amostras e o teste de Bartlett, indicava que as diferenças nos desvios padrão dos diferentes grupos experimentais, não eram significantivas (Zar, 1999).

\section{RESULTADOS}

\section{Contagem total de células}

Os animais do grupo LPS-C apresentaram número significativamente maior de células inflamatórias no lavado peritoneal em relação aos animais inoculados com SSE, confirmando a efetividade do estímulo com LPS. Os animais do grupo LPS-DZ10 apresentaram significativamente menos células no influxo inflamatório em relação ao grupo LPS-C, conforme apresentado na Tabela I.

\section{Contagem de mononucleares (MN) e polimorfonucleares (PMN)}

Os animais tratados com $10 \mathrm{mg} / \mathrm{kg}$ de diazepam apresentaram tendência à redução da porcentagem de células $\mathrm{MN}$, com aumento correspondente de PMN, independentemente do desafio, com diferença significativa em relação àqueles tratados com $1 \mathrm{mg} / \mathrm{kg}$ (Tabela I).

\section{TNF- $\alpha$}

Os animais tratados com $10 \mathrm{mg} / \mathrm{kg}$ de diazepam apresentaram redução estatisticamente significativa na atividade do TNF - $\alpha$ independentemente do tipo de inóculo (Figura 1).

\section{Espraiamento de Macrófagos}

Os animais tratados com 1 ou $10 \mathrm{mg} / \mathrm{kg}$ de diazepam e inoculados com LPS apresentaram proporção significativamente menor de macrófagos espraiados em relação àqueles não tratados. $\mathrm{O}$ tratamento com diazepam não alterou a porcentagem de macrófagos espraiados nos animais inoculados com SSE, ou seja, não estimulados (Figura 2). 
TABELA I - Número total de leucócitos e porcentagem relativa de MN e PMN no lavado peritoneal 16 horas após a inoculação intraperitoneal de LPS ou SSE em camundongos previamente tratados ou não com $1 \mathrm{ou} 10 \mathrm{mg} / \mathrm{kg}$ de diazepam

\begin{tabular}{|c|c|c|c|c|c|c|}
\hline \multirow{2}{*}{$\begin{array}{l}\text { GRUPO } \\
\text { TRATAMENTO }\end{array}$} & \multicolumn{3}{|c|}{ SSE } & \multicolumn{3}{|c|}{ LPS } \\
\hline & $\mathrm{C}$ & DZ1 & DZ10 & C & DZ1 & DZ10 \\
\hline Total de células $\left(* 10^{5}\right)$ & $22 \pm 2,04^{\mathrm{a}}$ & $21 \pm 2,21^{\mathrm{a}}$ & $22 \pm 1,81^{a}$ & $37 \pm 5,37^{b}$ & $30 \pm 1,87 \mathrm{bc}$ & $27 \pm 1,25^{c}$ \\
\hline$\% \mathrm{MN}$ & $70 \pm 4,15^{\mathrm{a}}$ & $72 \pm 3,30^{\mathrm{a}}$ & $54 \pm 1,95^{b}$ & $26 \pm 4,04^{\mathrm{cd}}$ & $29 \pm 3,74$ ce & $15 \pm 1,67 \mathrm{df}$ \\
\hline$\% \mathrm{PMN}$ & $30 \pm 4,15^{\mathrm{a}}$ & $28 \pm 3,30^{\mathrm{a}}$ & $46 \pm 1,95^{b}$ & $74 \pm 4,04^{\mathrm{cd}}$ & $71 \pm 3,74^{\text {ce }}$ & $85 \pm 1,67 \mathrm{df}$ \\
\hline
\end{tabular}

Letras iguais em colunas diferentes - não significativo.

Letras diferentes em colunas diferentes - significativo $(\mathrm{p}<0,05)$.

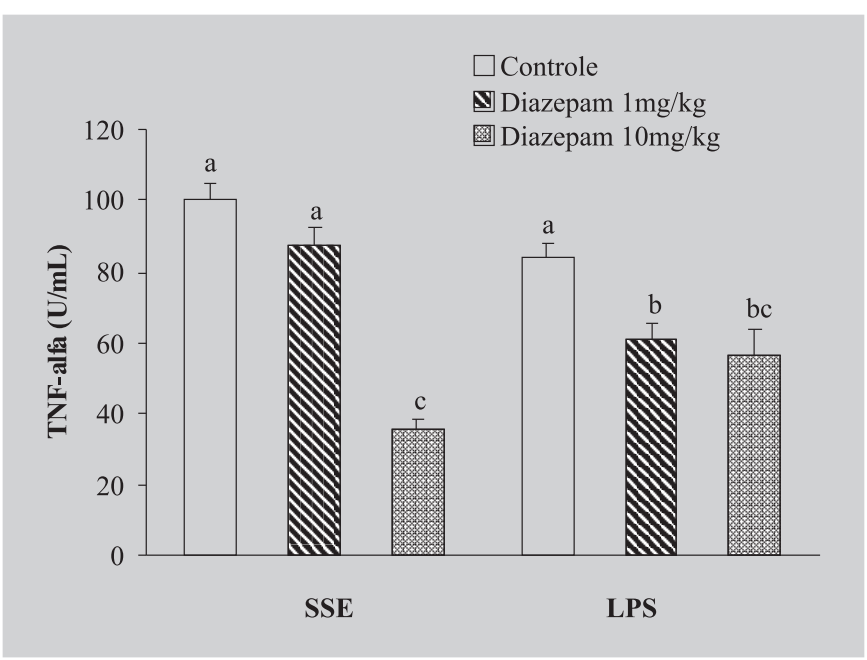

FIGURA 1 - Atividade do TNF- $\alpha$ no lavado peritoneal 16 horas após a inoculação intraperitoneal de LPS ou SSE em camundongos previamente tratados ou não com 1 ou $10 \mathrm{mg} / \mathrm{kg}$ de diazepam. Letras iguais em colunas diferentes - não significativo. Letras diferentes em colunas diferentes - significativo $(\mathrm{p}<0,05)$.

\section{DISCUSSÃO}

Após a administração intraperitoneal de diazepam em ratos, a droga é rapidamente absorvida e atinge uma concentração máxima no plasma e no fluido cerebroespinal em aproximadamente 10 minutos, mantendo-se elevada até uma hora depois (acima de 500 e $200 \mathrm{ng} / \mathrm{mL}$, respectivamente) e caindo posteriormente a níveis muito baixos, com redução gradativa dos efeitos clínicos (Friedman et al., 1986; Klotz et al., 1976a; Walker et al., 1998). Marc e Morselli (1969) demonstraram que até 60 minutos após a administração intraperitoneal de diazepam, os efeitos da droga são evidentes e os níveis plasmáticos de corticosterona são altos, decaindo rapidamente uma hora depois.

Portanto, o desafio com LPS uma hora após a administração subcutânea de diazepam garantiu a persistência da ação central e periférica da droga durante o desenvolvi-

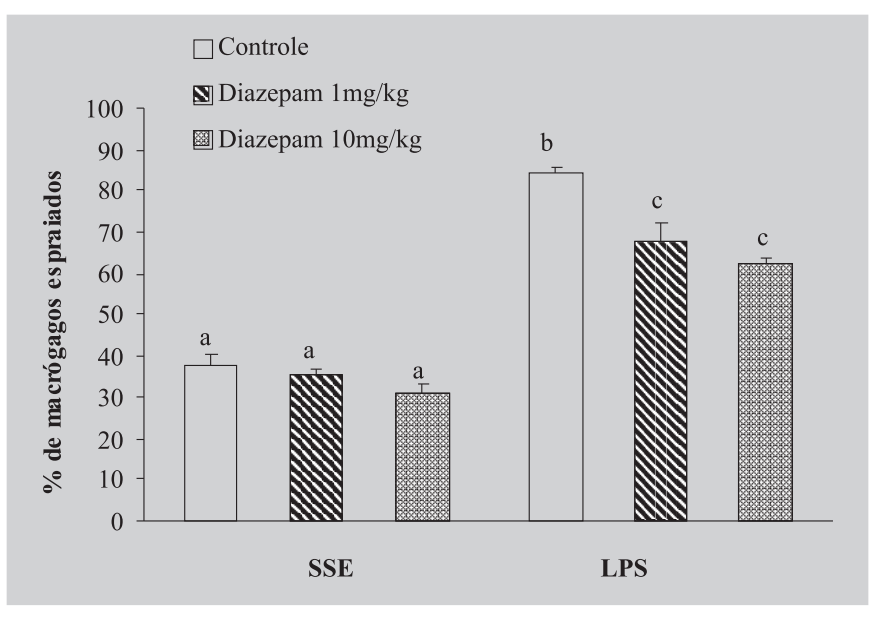

FIGURA 2 - Porcentagem de macrófagos espraiados no lavado peritoneal 16 horas após a inoculação intraperitoneal de LPS ou SSE em camundongos previamente tratados ou não com 1 ou $10 \mathrm{mg} / \mathrm{kg}$ de diazepam. Letras iguais em colunas diferentes - não significativo. Letras diferentes em colunas diferentes significativo $(p<0,05)$.

mento do processo inflamatório, considerando que a absorção via intramuscular ou subcutânea é mais lenta que a intraperitoneal (Garzon, 1999).

Por outro lado, Perecic et al. (1984) demonstraram experimentalmente que os níveis de corticosterona no plasma podem variar dependendo da dose e do benzodiazepínico utilizado, podendo influenciar o efeito imunomodulador dessas drogas. Além disso, o tempo de meia vida plasmática do diazepam é substancialmente menor em ratos do que no homem (Klotz et al., 1976b).

Em razão desta diferença na farmacocinética da droga, doses de 10 e $20 \mathrm{mg}$ de diazepam $/ \mathrm{kg}$ de peso administradas a ratos equivalem a aproximadamente 0,2 e $0,3 \mathrm{mg} / \mathrm{kg}$ no homem, indicadas para o tratamento da epilepsia (Walker et al., 1998). Por este motivo, optamos por utilizar neste trabalho, doses de diazepam de 1 e $10 \mathrm{mg} / \mathrm{kg}$ de peso, na tentativa de compararmos a influência de duas 
diferentes doses sobre a resposta inflamatória aguda, ambas dentro do limite de inclusão de doses utilizadas no tratamento humano.

Em experimentos-piloto realizados previamente, concluímos que o melhor tempo de influxo inflamatório encontrava-se em torno de 16 horas após o estímulo com LPS onde havia um pico de células inflamatórias na cavidade peritoneal e intensa migração de neutrófilos, permitindo avaliar experimentalmente qualquer possível influência do tratamento sobre esses parâmetros.

No presente trabalho, a redução significativa do número total de leucócitos no lavado peritoneal dos animais tratados com $10 \mathrm{mg}$ de diazepam $/ \mathrm{kg}$ de peso, é compatível com os resultados obtidos por Lazzarini et al. $(2001,2003)$ que observaram que o diazepam, em dose única de $10 \mathrm{mg} / \mathrm{kg}$ de peso por via i.p., induz redução significativa do edema inflamatório plantar e do número total de leucócitos no exsudado pleural induzido por carragenina em ratos.

Por outro lado, a redução significativa da produção de TNF- $\alpha$ após o tratamento com diazepam $(10 \mathrm{mg} / \mathrm{kg}$ de peso) parece confirmar os resultados obtidos por Zavala et al. (1990), que demonstraram que injeções intraperitoneais de BZD inibem significativamente, após 48 horas, a produção de TNF- $\alpha$, IL-1 e IL-6 induzida por LPS em macrófagos peritoneais de camundongos.

Bidri et al. (1999) relataram também que os BZDs diazepam e midazolam inibem, in vitro e de forma dosedependente, a secreção de TNF- $\alpha$ em culturas de mastócitos obtidos de camundongos. Este efeito, potencialmente relevante para o desenvolvimento da resposta antiinfecciosa, pode estar relacionado a possível modulação, induzida por diazepam, dos receptores periféricos para benzodiazepínicos, conforme proposto por outros autores (Torres et al., 2000; Zavala et al., 1985, 1990).

Tem sido demonstrado em camundongos que a produção de TNF- $\alpha$ é máxima 2 a 4 horas após estimulação de macrófagos pulmonares e peritoneais com LPS (Nelson et al., 1989; Zhang, Morrison, 1993). Esta cinética poderia explicar as concentrações equivalentes de TNF- $\alpha, 16$ horas após o estímulo, nos animais dos grupos não tratados independente do estímulo.

Com relação à função macrofágica, o espraiamento celular é um processo bioquímico complexo que envolve interações entre proteínas de membrana, permitindo a aderência e estabilização da célula no substrato, sendo considerado como um sinal morfológico inicial do processo de ativação de macrófagos (Oliveira, 1994).

Neste contexto, Martino et al. (1995) mostraram que doses terapêuticas de diazepam ( $7 \mu \mathrm{g} /$ camundongo) foram capazes de modular o conteúdo de actina em macrófagos, num efeito dose-dependente. Também Massoco \& Palermo-Neto
(1999) demonstraram que o diazepam $(1,5 \mathrm{mg} / \mathrm{kg})$, quando administrado intraperitonealmente em camundongos, durante sete dias, induz redução do índice de espraiamento de macrófagos peritoneais estimulados por BCG.

Portanto, os resultados obtidos no presente trabalho reforçam aqueles descritos por outros autores e sugerem que a menor porcentagem de macrófagos espraiados observada nos animais tratados com diazepam pode também estar relacionada à modulação de RPBs, os quais são densamente expressos por macrófagos (Zavala et al., 1985), interferindo com o processo de reorganização de microfilamentos de actina em macrófagos, que é um evento chave no primeiro estágio do processo de fagocitose.

\section{CONCLUSÕES}

Os resultados do presente estudo confirmam e estendem os conhecimentos sobre a imunofarmacologia do diazepam, indicando que este agente ansiolítico pode deprimir significativamente o influxo de leucócitos, o espraiamento de macrófagos peritoneais e a liberação de TNF- $\alpha$, após estimulação com LPS em camundongos, embora sem influenciar significativamente a cinética do influxo celular de $\mathrm{MN}$ e PMN no foco inflamatório peritoneal. Esses achados corroboram a hipótese de que o tratamento com diazepam, em dose única, pode influenciar de forma significativa no desenvolvimento da resposta inflamatória aguda induzida por endotoxinas, com possíveis implicações na eficiência da resposta antiinfecciosa.

\section{ABSTRACT}

\section{Diazepam, in a single dose, inhibits cellular chemotaxis, macrophage stimulation, and TNF- $\alpha$ activity in LPS- induced acute inflammatory responses in mice}

Benzodiazepines are one of the most frequently prescribed drugs due to their anxiolytic properties. The aim of this study was to evaluate the effects of diazepam on lipopolysaccharide-induced peritoneal acute inflammatory responses. Swiss mice were treated with diazepam in a single dose of 1 or $10 \mathrm{mg} / \mathrm{kg}$ - subcutaneously $1 \mathrm{~h}$ before an intraperitoneal injection of lipopolysaccharide or sterile saline solution. The mice were killed $16 \mathrm{~h}$ after and the cells were washed from the peritoneal cavity to determine the total number of cells and the mononuclear and polimorfonuclear subpopulations, as well as the TNF-alpha activity and percentage of spread macrophages. Our results showed that the diazepam treatment (1 and $10 \mathrm{mg} / \mathrm{kg}$ ) induced a significant reduction in the LPS-induced macrophage stimulation and TNF- $\alpha$ activity. Diazepam 
$(10 \mathrm{mg} / \mathrm{kg})$ also reduced the inflammatory cellular migration when compared to the control. It can be concluded that the diazepam treatment in a single dose is able to influence the inflammatory cellular influx, macrophage stimulation and $T N F-\alpha$ activity in the acute inflammatory response in mice, having possible implications on the anti-infectious response efficiency.

UNITERMS: Diazepam. Lipopolysaccharide. Acute inflammatory response. TNF- $\alpha$. Cellular migration/ inhibition. Macrophages/stimulation.

\section{AGRADECIMENTOS}

Ao $\mathrm{CNPq}$, pelo suporte financeiro e a Ivete D.P. Palombo, Zélia M.S. Venézio e Marisa Campos Polesi Placeres, pelo auxílio técnico.

\section{REFERÊNCIAS BIBLIOGRÁFICAS}

AMURA, C.R.; CHEN, L.C.; HIROHASHI, N.; LEI, M.G.; MORRISON, D.C. Two functionally independent pathways for lipopolysaccharide-dependent activation of mouse peritoneal macrophages. J. Immunol., v.159, p.5079-5083, 1997.

BIDRI, M.; ROYER, B.; AVERLANT, G.; BISMUTH, G.; GUILLOSSON, J.J.; AROCK, M. Inhibition of mouse mast cell proliferation and proinflammatory mediator release by benzodiazepines. Immunopharmacology, v.43, n.1, p.75-86, 1999.

BRATTIG, N.B.W.; GARZA, C.M.; TISCHENDORF, F.W. Improved Randolph stain for direct leukocyte differentiation and determination of total eosinophil count in a hemocytometer. Biotech. Histochem., v.63, p.255$259,1993$.

CARLOS, I.Z.; ZINI, M.M.C.; SGARBI, D.B.G.; ANGLUSTER, J.; ALVIANO C.S.; SILVA, C. L. Disturbance in the production of interleukin-1 and tumor necrosis factor in disseminated murine sporotrichosis. Mycopathologia, v.127, p.189-194, 1994.

FINNERTY, M.; MARCZYNSKI, T.J.; AMIRAULT, H. J.; URBANCIC, M.; ANDERSEN, B.R. Benzodiazepines inhibit neutrophil chemotaxis and superoxide production in a stimulu dependent manner, PK-11195 antagonizes these effects. Immunopharmacology, v.22, p.185-193, 1991.
FRIEDMAN, H.; ABERNETHY, D.R.; GREENBLATT, D.J.; SHADER, R.I. The pharmacokinetics of diazepam and desmethyldiazepam in rat brain and plasma. Psychopharmacology, v.88, p.267-270, 1986.

GALDIERO F.; BENTIVOGLIO, C.; NUZZO, I.; IANIELLO, R.; CAPASSO, C.; MATTERA, S.; NAZZARO, C.; GALDIERO, M.; CARRATELLI, C.R. Effects of benzodiazepines on immunodeficiency and resistance in mice. Life Sci., v.57, p.2413-2423, 1995.

GARZON, E. Benzodiazepínicos - de ansiolíticos a antiepiléticos. In:YACUBIAN, E.M.T.(Ed.) Tratamento medicamentoso da epilepsias. São Paulo: Lemos Editorial, 1999. p.92-104.

GAVISH, M.; BACHMAN, I.; SHOUKRUN, R.; KATZ, Y.; VEENMAN, L.; WEISINGER, G.; WEIZMAN, A. Enigma of the peripheral benzodiazepine receptor. Pharmacol. Rev., v.51, n.4, p.629-650, 1999.

HIRSCH, J. B.; BEYER, C. F.; MALKOWITZ, L.; BEER, B.; BLUME, A. J. Mitochondrial benzodiazepine receptors mediate inhibition of mitochondrial respiratory control. Mol. Pharmacol., v.35, p.157-163, 1989.

KLOTZ, U.; ANTONIN, K. H.; BIECK, P. R. Comparison of the pharmacokinetics of diazepam after single and subchronic doses. Eur. J. Clin. Pharmacol., v.10, p.121126, 1976a.

KLOTZ, U.; ANTONIN, K.H.; BIECK, P. R. Pharmacokinetics and plasma binding of diazepam in man, dog, rabbit, guinea pig and rat. J. Pharmacol. Exp. Ther., v.199, n.1, p.67-73, 1976b.

LAZZARINI, R.; MALUCELLI, B. E.; MUSCARA, M. N.; NUCCI, G.; PALERMO-NETO, J. Reduction of inflammation in rats by diazepam: tolerance development. Life Sci., v.72, p.2361-2368, 2003.

LAZZARINI, R.; MALUCELLI, B.E.; PALERMO-NETO, $\mathrm{J}$. Reduction of acute inflammation in rats by diazepam: role of peripheral benzodiazepine receptors and corticosterone. Immunopharmacol. Immunotoxicol., v.23, n.2, p.253-265, 2001.

MARC V.; MORSELLI, P.L. Effect of diazepam on plasma corticosterone levels in the rat. J. Pharm. Pharmacol., v.21, p.784-785, 1969. 
MARTINO, L.D.; NAZZARO, C.; MERONE, A.; CONCILIO, S.; GALDIERO, M. Modification of actin in peritoneal macrophages after diazepam treatment. Immunopharmacol. Immunotoxicol., v.17, n.3, p.551564, 1995.

MASSOCO, C.O.; PALERMO-NETO, J. Diazepam effects on peritoneal macrophage activity and corticosterone serum levels in balb/c mice. Life Sci., v.65, n.20, p.21572165, 1999.

MASSOCO, C.O.; PALERMO-NETO, J. Effects of midazolam on equine innate immune response: a flow cytometric study. Vet. Immunol. Immunopathol., v.95, p.11-19, 2003.

McCARRON, R.M.; GOROFF, D.K.; LUHR, J. E.; MURPHY, M.A.; HERSCOWITZ, H.B. Methods for the collection of peritoneal and alveolar macrophages. Methods Enzymol., v.108, p.274-284, 1984.

NELSON, S.; BAGBY, G. J.; BAINTON, B.G.; WILSON, L.A.; THOMPSON, J.J.; SUMMER, W.R. Compartimentalization of intraalveoalar and systemic lipopolysaccharide- induced tumor necrosis factor and the pulmonary inflammatory response. J. Infect. Dis., v.159, n.2, p.189-194, 1989.

OLIVEIRA, C.A. Estudo sobre a indução da fagocitose de imunocomplexos de C3b ou gM homóloga por macrófagos peritoneais de camundongos. Ribeirão Preto, 1994. 161 p. [Dissertação de Mestrado. Faculdade de Medicina. Universidade de São Paulo].

PAPADOPOULOS, V.; MUKHIN, G.; COSTA, F.; KRUEGER, K.E. The peripheral type benzodiazepine receptor is functionally linked to leydig cell steroidogenesis. J. Biol. Chem., v.265, p.3772-3779, 1990 .

PERICIC, D.; LAKIC, N.; MANEV, H. Effect of diazepam on plasma corticosterone levels. Psychopharmacology, v.83, p.79-81, 1984.

PINTO, F.G. Pesquisa de citocinas, produção de óxido nítrico e avaliação funcional de macrófagos pulmonares na pneumonite de hipersensibilidade induzida em camundongos pelo Paracoccidioides brasiliensis. Botucatu, 2000. 107 p. [Tese de Doutorado. Faculdade de Medicina. Universidade Estadual Paulista].
RABINOVITCH, M.; MANEJIAS, R.E.; RUSSO, M.; ABBEY, E.E. Increased spreading of macrophages from mice treated with interferon inducers macrophage spreading in vitro. Cell. Immunol., v.29, p.86-95, 1977.

RUFF, M.R.; PERT, C.B.; WEBER, R.J.; WHAL, L.M; WHAL, L.M.; PAUL, S.M. Benzodiazepine receptormediated chemotaxis of human monocytes. Science, v.229, p.1281-1283, 1985.

RUIZ, I.; OFFERMANNS, J.; LANCTOT, K.L.; BUSTO, U. Comparative study on benzodiazepine use in Canada and Chile. J. Clin. Pharmacol., v.33, p.124-125, 1993.

SCHLUMPF, M.; LICHTENSTEIGER, W.; VAN LOVEREN, H. Impaired host resistance to Trichinella spiralis as a consequence of prenatal treatment of rats with diazepam. Toxicology, v.94, p.223-230, 1994.

SCHLUMPF, M.; PARMAR, R.; RAMSEIER, H.R.; LICHTENSTEIGER, W. Prenatal benzodiazepine immunodepression: possible involvement of a peripheral benzodiazepine site. Dev. Pharmacol. Ther., v.15, p.178$85,1990$.

SCHREIBER, A.A.; FREI, K.; LICHTENSTEIGER, W.; SHULUMPF, M. Alterations in interleukin 6 production by LPS and Com A stimulated mixed splenocytes, spleen macrophages and lymphocytes in prenatally - diazepam - exposed rats. Agents Actions, v.39, p.166-173, 1993.

SILVA, F.R.; PALERMO-NETO, J. Developmental, neuro and immunotoxic effects of perinatal diazepam treatment in rats. Immunopharmacol. Immunotoxicol., v.21, p.247$265,1999$.

TORRES, S.R.; FRÖDE, T.S.; NARDI, G.M.; VITA, N.; REEB, R.; FERRARA, P.; RIBEIRO-DO-VALE, R.M.; FARGES, R.C. Anti-inflammatory effects of peripheral benzodiazepine receptor ligands in two mouse models of inflammation. Eur. J. Pharmacol., v.408, p.199-211, 2000 .

WALKER, M.C.; TONG, X.; BROWN, S.; SHORVON, S.D.; PATSALOS, P.N. Comparison of single- and repeated-dose pharmacokinetics of diazepam. Epilepsia, v.39, n.3, p.283-289, 1998.

WANG, J.K.; MORGAN， J.I.; SPECTOR, S. Benzodiazepines that bind at peripheral sites inhibit cell proliferation. Proc. Natl. Acad. Sci. USA, v.81, p.753756, 1984. 
ZAR, J.H. Biostatistical analysis. 4. ed. Prentice Hall: Upper Saddle River, 1999. 942 p.

ZAVALA, F. Benzodiazepines, anxiety and immunity. Pharmacol. Ther., v.75, n.3, p.199-216, 1997.

ZAVALA, F.; HAUMONT, J.; LENFANT, M. Interactions of benzodiazepines with mouse macrophages. Eur. J. Pharmacol., v.10, p.561-566, 1985.
ZAVALA, F.; TAUPIN, V.; DESCAMPS-LATSCHA, B. In vivo treatment with benzodiazepines inhibits murine phagocyte oxidative metabolism and production of interleukin 1, tumor necrosis factor and interleukin-6.J. Pharmacol. Exp. Ther., v.255, n.2, p.442-450, 1990.

ZHANG, X.; MORRISON, D.C. Lipopolysaccharideinduced selective priming effects on tumor necrosis factoralfa and nitric oxide production in mouse peritoneal macrophages. J. Exp. Med., v.177, p.511-516, 1993.

Recebido para publicação em 09 de novembro de 2006 Aceito para publicação em 04 de agosto de 2008 